\title{
Glivenko-Cantelli Properties of some Generalized Empirical DF's and Strong Convergence of Generalized $L$-Statistics *
}

\author{
R. Helmers ${ }^{1}$, P. Janssen ${ }^{2}$ and R. Serfling ${ }^{3}$ \\ ${ }^{1}$ Centre for Mathematics and Computer Science, Kruislaan 413, SJ-1098 Amsterdam, \\ The Netherlands \\ ${ }^{2}$ Limburgs Universitair Centrum, B-3610 Diepenbeek, Belgium \\ ${ }^{3}$ Department of Mathematical Sciences, Johns Hopkins University, Baltimore, MD 21218, USA
}

\begin{abstract}
Summary. We study a nonclassical form of empirical $d f H_{n}$ which is of $U$ statistic structure and extend to $H_{n}$ the classical exponential probability inequalities and Glivenko-Cantelli convergence properties known for the usual empirical $d f$. An important class of statistics is given by $T\left(H_{n}\right)$, where $T(\cdot)$ is a generalized form of $L$-functional. For such statistics we prove almost sure convergence using an approach which separates the functional-analytic and stochastic components of the problem and handles the latter component by application of Glivenko-Cantelli type properties. Classical results for $U$ statistics and $L$-statistics are obtained as special cases without addition of unnecessary restrictions. Many important new types of statistics of current interest are covered as well by our result.
\end{abstract}

\section{Introduction}

Let $X_{1}, \ldots, X_{n}$ be independent r.v.'s having common $d f F$ and let $h$ be a measurable function from $\mathbb{R}^{m}$ to $\mathbb{R}$. Define the associated $d f H_{F}(y)=P_{F}\left\{h\left(X_{1}, \ldots, X_{m}\right)\right.$ $\leqq y\}, y \in \mathbb{R}$ and empirical $d f$

$$
H_{n}(y)=n_{(m)}^{-1} \sum 1\left\{h\left(X_{i_{1}}, \ldots, X_{i_{m}}\right) \leqq y\right\}, \quad y \in \mathbb{R},
$$

where the sum is taken over all $n_{(m)}=n(n-1) \ldots(n-m+1) m$-tuples $\left(i_{1}, \ldots, i_{m}\right)$ of distinct elements from $\{1, \ldots, n\}$. For each fixed $y, H_{n}(y)$ is a $U$-statistic with mean $H_{F}(y)$; in the case $m=1$ and $h(x)=x, H_{n}$ reduces to the usual empirical df $F_{n}$.

Statistics of the form $T\left(H_{n}\right)$ were investigated by Serfling (1984) for $T(\cdot)$ an $L$-functional and by Janssen et al. (1984) for $T(\cdot)$ a more general type of $L$-functional. Certain Glivenko-Cantelli properties for $H_{n}$ and asymptotic normality results for $T\left(H_{n}\right)$ were established.

* Research supported by the U.S. Department of Navy under Office of Naval Research Contract No. N00014-79-C-0801 and by NATO under Research Grant No. 0034/87 
The present paper provides further Glivenko-Cantelli results for the generalized empirical $d f H_{n}$ (Sect. 2) and strong convergence results for the "generalized $L$-statistics" discussed above (Sects. 3 and 4). Results for the multi-sample case are indicated also.

Our key tool in establishing Glivenko-Cantelli results is an extension to $H_{n}$ of the exponential probability inequality of Dvoretzky et al. (1956) for the Kolmogorov distance between $F_{n}$ and $F$. Our strong convergence results generalize those of Hoeffding (1961) for $U$-statistics and of van Zwet (1980) for $L$ statistics, without adding unnecessary restrictions.

In our treatment of the strong convergence problem for generalized $L$-statistics, we separate the functional-analytic and stochastic components of the problem. As a result, one can obtain results for statistics $T\left(H_{n}\right)$ by substituting $H_{n}$ for $G_{n}$ into basic convergence theorems proved for the functional $T(\cdot)$ defined on deterministic sequences $\left\{G_{n}\right\}$. This permits flexibility and easier generalization in the choice of (nonclassical) empirical $d f$ to be used and illuminates the fundamental issues involved in the convergence problem.

It should be noted that results on the behavior of $H_{n}$ typically apply also to the empirical $d f H_{F_{n}}$ given by putting $F_{n}$ for $F$ in the definition of $H_{F}$. For each fixed $y, H_{F_{n}}(y)$ is a von Mises statistic.

The closeness of $H_{n}$ and $H_{F_{n}}$ may be evaluated through the relations

$$
n^{m}\left(H_{n}-H_{F_{n}}\right)=\left(n^{m}-n_{(m)}\right)\left(H_{n}-\tilde{H}_{n}\right),
$$

where $\tilde{H}_{n}(y)$ is the average of terms $1\left\{h\left(X_{i_{1}}, \ldots, X_{i_{m}}\right) \leqq y\right\}$ with at least one equality $i_{a}=i_{b}, a \neq b$, and

$$
n^{m}-n_{(m)}=O\left(n^{m-1}\right) .
$$

We shall leave results for $H_{F_{n}}$ implicit from those stated for $H_{n}$.

\section{Glivenko-Cantelli Results}

Here we establish results on the almost sure convergence to 0 of $\left\|H_{n}-H_{F}\right\|_{\infty}$, where $\|f\|_{\infty}$ denotes sup $|f(\mathrm{x})|$. Our first result is an analogue of the exponential probability inequality of Dvoretzky, Kiefer and Wolfowitz (1956) for $\left\|F_{n}-F\right\|_{\infty}$. Their inequality, which serves as a lemma in our development, is the following.

Lemma 2.1. There exists a finite constant $C_{0}$, not depending on $F$, such that

$$
P\left\{\left\|F_{n}-F\right\|_{\infty}>d\right\} \leqq C_{0} \exp \left\{-2 n d^{2}\right\}, \quad d>0, n \geqq 1 .
$$

We shall utilize Lemma 2.1 to establish a related inequality for the momentgenerating function of $\left\|F_{n}-F\right\|_{\infty}$, which is evidently a novel result for the classical empirical $d f$.

Lemma 2.2. There exists a finite constant $C_{1}$, not depending on $F$, such that

$$
E\left\{\exp s\left\|F_{n}-F\right\|_{\infty}\right\} \leqq\left(1+C_{1} s n^{-1 / 2}\right) \exp \left\{s^{2} / 8 n\right\}, \quad s>0, n \geqq 1 .
$$


Proof. By a well-known identity and an application of Lemma 2.1, we have

$$
\begin{aligned}
E\left\{\exp s\left\|F_{n}-F\right\|_{\infty}\right\} & =\int_{0}^{\infty} P\left\{\exp \left(s\left\|F_{n}-F\right\|_{\infty}\right)>t\right\} d t \\
& =1+\int_{1}^{e^{s}} P\left\{\left\|F_{n}-F\right\|_{\infty}>(\log t) / s\right\} d t \\
& \leqq 1+C_{0} \int_{1}^{e^{s}} \exp \left\{-2 n(\log t)^{2} / s^{2}\right\} d t
\end{aligned}
$$

where we have used the fact that $0 \leqq\left\|F_{n}-F\right\|_{\infty} \leqq 1$. The integral in (2.3) is easily evaluated as (here $N\left(\mu, \sigma^{2}\right)$ denotes a normal distribution with mean $\mu$ and variance $\sigma^{2}$ )

$$
\left(\frac{\pi}{2}\right)^{1 / 2} s n^{-1 / 2} P\left\{0 \leqq N\left(s^{2} / 4 n, s^{2} / 4 n\right) \leqq s\right\} \exp \left\{s^{2} / 8 n\right\},
$$

so that (2.2) follows with $C_{1}=C_{0}\left(\frac{\pi}{2}\right)^{1 / 2}$.

We now extend Lemma 2.2 to the empirical $d f H_{n}$.

Lemma 2.3. For the constant $C_{1}$ in (2.2), we have

$$
E\left\{\exp s\left\|H_{n}-H_{F}\right\|_{\infty}\right\} \leqq\left(1+C_{1} s[n / m]^{-1 / 2}\right) \exp \left\{s^{2} / 8[n / m]\right\}, \quad s>0, n \geqq m .
$$

(Here $[\cdot]$ denotes greatest integer part.)

Proof. Utilizing a representation for $U$-statistics given by Hoeffding (1963) (or see Serfling (1980), p. 180), we have the representation

$$
H_{n}-H_{F}=\frac{1}{n !} \sum_{i=1}^{n !}\left(H_{n}^{(i)}-H_{F}\right)
$$

where each $H_{n}^{(i)}$ is a classical empirical $d f$ based on $[n / m]$ terms $h\left(X_{i_{1}}, \ldots, X_{i_{m}}\right)$, $h\left(X_{i_{m+1}}, \ldots, X_{i_{2 m}}\right), \ldots$ corresponding to a particular permutation $\left(i_{1}, i_{2}, \ldots, i_{n}\right)$ of $(1, \ldots, n)$. By convexity of the exponential function and Lemma 2.2 , we obtain (2.4).

Applying Lemma 2.3 in connection with the elementary relation

$$
P\left\{\left\|H_{n}-H_{F}\right\|_{\infty}>d\right\} \leqq e^{-s d} E\left\{e^{s\left\|H_{n}-H_{F}\right\|_{\infty}}\right\}, \quad s>0,
$$

and with $s=4[n / m] d$, we obtain 
Theorem 2.1. There exists a finite constant $C_{1}$, not depending on $F$, or $h$, such that

$$
P\left\{\left\|H_{n}-H_{F}\right\|_{\infty}>d\right\} \leqq\left(1+4 C_{1}[n / m]^{1 / 2} d\right) \exp \left\{-2[n / m] d^{2}\right\}, \quad d>0, n \geqq m .
$$

It should be noted that Theorem 2.1, specialized to the case $m=1$, is not as sharp as Lemma 2.1. This is due to the indirect method of proof using moment-generating functions and perhaps also due to a lack of sharpness in the bound obtained in Lemma 2.2. Nevertheless, for practical purposes in typical applications, Theorem 2.1 in its present form is sufficiently powerful. For example, it immediately yields, via the Borel-Cantelli lemma, the following rate for the almost sure convergence of $\left\|H_{n}-H_{F}\right\|_{\infty}$ to 0 .

Corollary 2.1. There exists a finite constant $C_{m}$, not depending on $F$ or otherwise on $h$, such that with probability 1

$$
\limsup _{n \rightarrow \infty}\left(\frac{n}{\log n}\right)^{1 / 2}\left\|H_{n}-H_{F}\right\|_{\infty}<C_{m} .
$$

(For example, $C_{m}>(m / 2)^{1 / 2}$ suffices.) In the case $m=1$, it follows by the LIL for $\left\|F_{n}-F\right\|_{\infty}$ (due to Chung (1949); see also Serfling (1980), p. 62, for discussion) that the limsup in $(2.8)$ remains finite with $(\log n)$ replaced by $(\log \log n)$. Such refinements of (2.8) involve tools more delicate than Lemma 2.1 and Theorem 2.1 and will not be pursued here.

Our next result treats the almost sure behavior of weighted discrepancies between $H_{n}$ and $H_{F}$, extending the strengthened Glivenko-Cantelli theorem for $F_{n}$ given by Wellner (1977).

Theorem 2.2. Let $q$ be a nonnegative continuous function in $[0,1]$ which is nondecreasing in $[0, \delta]$ and nonincreasing in $[1-\delta, 1]$, for some $\delta>0$, and satisfies $\int_{0}^{1}[q(t)]^{-1} d t<\infty$. Then with probability 1

$$
\left\|\left(H_{n}-H_{F}\right) / q \circ H_{F}\right\|_{\infty} \rightarrow 0, \quad n \rightarrow \infty .
$$

Proof. We follow the technique of Wellner (1977). Let $\varepsilon>0$ be given and choose $M<\delta$ such that $\int_{0}^{M}[q(t)]^{-1} d t<\varepsilon$ and $\int_{1-M}^{1}[q(t)]^{-1} d t<\varepsilon$ and such that $M$ and $1-M$ are continuity points of $H_{F}^{-1}$. Put $\theta_{0}=H_{F}^{-1}(M)$ and $\theta_{1}=H_{F}^{-1}(1-M)$. Now write

$$
\begin{aligned}
& \left\|\left(H_{n}-H_{F}\right) / q \circ H_{F}\right\|_{\infty} \leqq \sup _{y<\theta_{0}} \frac{H_{n}(y)}{q \circ H_{F}(y)}+\sup _{y<\theta_{0}} \frac{H_{F}(y)}{q \circ H_{F}(y)} \\
& \quad+\sup _{\theta_{0} \leqq y \leqq \theta_{1}}\left|\frac{H_{n}(y)-H_{F}(y)}{q \circ H_{F}(y)}\right|+\sup _{y>\theta_{1}} \frac{1-H_{n}(y)}{q \circ H_{F}(y)}+\sup _{y>\theta_{1}} \frac{1-H_{F}(y)}{q \circ H_{F}(y)} \\
& =A_{1}+A_{2}+A_{3}+A_{4}+A_{5} .
\end{aligned}
$$


Now

$$
A_{3} \leqq\left(\sup _{\theta_{0} \leqq y \leqq \theta_{1}}\left[q \circ H_{F}(y)\right]^{-1}\right)\left\|H_{n}-H_{F}\right\|_{\infty} \rightarrow 0 w p 1
$$

by Theorem 2.1. And, since $y<\theta_{0} \Rightarrow H_{F}(y)<M$, we have

$$
A_{2} \leqq \sup _{0<t<M} \frac{t}{q(t)} \leqq \sup _{0<t<M} \int_{0}^{t}[q(u)]^{-1} d u=\int_{0}^{M}[q(u)]^{-1} d u<\varepsilon
$$

Similarly, we have $A_{5}<\varepsilon$. And wp 1

$$
\begin{aligned}
A_{1} & \leqq n_{(m)}^{-1} \sum \sup _{y<\theta_{0}} \frac{1\left\{h\left(X_{i_{1}}, \ldots, X_{i_{m}}\right) \leqq y\right\}}{q \circ H_{F}(y)} \\
& \leqq n_{(m)}^{-1} \sum \frac{1\left\{h\left(X_{i_{1}}, \ldots, X_{i_{m}}\right) \leqq \theta_{0}\right\}}{q \circ H_{F}\left(h\left(X_{i_{1}}, \ldots, X_{i_{m}}\right)\right)} \rightarrow \int_{-\infty}^{\theta_{0}}\left[q \circ H_{F}(y)\right]^{-1} d H_{F}(y)<\varepsilon
\end{aligned}
$$

using the SLLN for $U$-statistics (Hoeffding (1961); or see Serfling (1980)) and the fact that

$$
\begin{aligned}
h\left(X_{i_{1}}, \ldots, X_{i_{m}}\right) \leqq y<\theta_{0} & \Rightarrow h\left(X_{i_{1}}, \ldots, X_{i_{m}}\right)<H_{F}^{-1}(M) \\
& \Rightarrow H_{F}\left(h\left(X_{i_{1}}, \ldots, X_{i_{m}}\right)\right)<M<\delta .
\end{aligned}
$$

Similarly, $A_{4}$ has an a.s. limit $<\varepsilon$.

Therefore, for every $\varepsilon>0$, $\limsup _{n \rightarrow \infty}\left\|\left(H_{n}-H_{F}\right) / q \circ H_{F}\right\|_{\infty}<5 \varepsilon$.

As an application of Theorem 2.2, we have

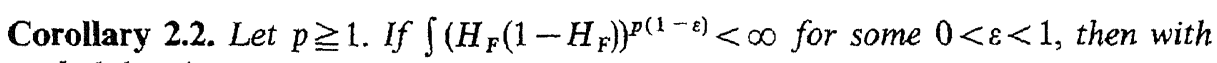
probability 1

$$
\left\|H_{n}-H_{F}\right\|_{p} \rightarrow 0, \quad n \rightarrow \infty
$$

$\left(\right.$ Here $\|g\|_{p}$ denotes $\left.\left(\int|g(x)|^{p} d x\right)^{1 / p}.\right)$

Proof. We write

$$
\left\|H_{n}-H_{F}\right\|_{p} \leqq\left\|\left(H_{n}-H_{F}\right) / q \circ H_{F}\right\|_{\infty} \cdot\left\|q \circ H_{F}\right\|_{p}
$$

and apply Theorem 2.2 with $q(t)=(t(1-t))^{1-\varepsilon}$.

It is easily checked that the assumption of Corollary 2.2 is satisfied if $E|h|^{p^{-1}+\delta}<\infty$ for some $\delta>0$ (cf. Lemma 2.2.1 of Helmers (1982)). 
Let $\mu_{n}$ and $\mu_{F}$ denote the probability measures on the class $\mathbf{B}$ of Borel sets in $\mathbb{R}$, associated with the $d f$ 's $H_{n}$ and $H_{F}$. Following Gaenssler (1983), for any subclass $\mathbf{C}$ of $\mathbf{B}$, we define the empirical $\mathbf{C}$-discrepancy

$$
D_{n}\left(\mathbf{C}, \mu_{F}\right):=\sup _{C \in \mathbf{C}}\left|\mu_{n}(C)-\mu_{F}(C)\right|
$$

and we assume that this quantity is Borel-measurable. Let $\mathbf{X}_{(n)}=\left(X_{n, 1}, \ldots, X_{n, n}\right)$ denote the vector of order statistics of $X_{1}, \ldots, X_{n}$.

Theorem 2.3. $\left\{D_{n}\left(\mathbf{C}, \mu_{F}\right)\right\}_{n \geq m}$ is a reverse submartingale with respect to the sequence of $\sigma$-fields $\mathbf{A}_{n}=\sigma\left\{\mathbf{X}_{(n)}, X_{n+1}, X_{n+2}, \ldots\right\}$, i.e., for $m \leqq k \leqq n$, with probability 1

$$
E\left\{D_{k}\left(\mathbf{C}, \mu_{F}\right) \mid \mathbf{A}_{n}\right\} \geqq D_{n}\left(\mathbf{C}, \mu_{F}\right) .
$$

Proof. Use the reverse martingale property (Serfling (1980), p. 180) of the $U$ statistic $\mu_{n}(C)-\mu_{F}(C)$, for each fixed $C \in \mathbf{C}$, to get

$$
E\left\{D_{k}\left(\mathbf{C}, \mu_{F}\right) \mid \mathbf{A}_{n}\right\} \geqq \sup _{C \in \mathbf{C}}\left|E\left\{\left(\mu_{k}(C)-\mu_{F}(C)\right) \mid \mathbf{A}_{n}\right\}\right|=D_{n}\left(\mathbf{C}, \mu_{F}\right) .
$$

In the case $m=1$ this reduces to Lemma 5 of Gaenssler (1983).

We now examine extensions to the multi-sample case. Consider $c$ independent collections of independent observations $\left\{X_{1}^{(1)}, \ldots, X_{n_{1}}^{(1)}\right\}, \ldots,\left\{X_{1}^{(c)}, \ldots, X_{n_{c}}^{(c)}\right\}$ taken from $d f^{\prime}$ 's $F^{(1)}, \ldots, F^{(c)}$, respectively.

Let a kernel $h\left(x_{1}^{(1)}, \ldots, x_{m_{1}}^{(1)} ; \ldots ; x_{1}^{(c)}, \ldots, x_{m_{c}}^{(c)}\right)$ mapping $\mathbb{R}^{m_{1}+\ldots+m_{c}}$ to $\mathbb{R}$ be given, put $F=\left(F^{(1)}, \ldots, F^{(c)}\right)$, denote by $H_{F}$ the $d f$ of $h\left(X_{1}^{(1)}, \ldots, X_{m_{1}}^{(1)} ; \ldots ; X_{1}^{(c)}, \ldots, X_{m_{c}}^{(c)}\right)$, and define the relevant empirical $d f$ by

$$
H_{z}(y)=\left[\prod_{j=1}^{c}\left(n_{j}\right)_{\left(m_{j}\right)}\right]^{-1} \sum 1\left\{h\left(X_{i_{11}}^{(1)}, \ldots, X_{i_{1 m_{1}}}^{(1)} ; \ldots ; X_{i_{c 1}}^{(c)}, \ldots, X_{i_{c m_{c}}}^{(c)}\right) \leqq y\right\},
$$

$y \in \mathbb{R}$, where $\underset{n}{n}=\left(n_{1}, \ldots, n_{c}\right)$ and the sum is taken over all $\left(n_{j}\right)_{\left(m_{j}\right)}=n_{j}\left(n_{j}-1\right) \ldots\left(n_{j}\right.$ $\left.-m_{j}+1\right) m_{j}$-tuples $\left(i_{j 1}, \ldots, i_{j m_{j}}\right)$ of distinct elements from $\left\{1, \ldots, n_{j}\right\}, 1 \leqq j \leqq c$. Finally, put $k_{\mathfrak{n}}=\min \left\{\left[n_{1} / m_{1}\right], \ldots,\left[n_{c} / m_{c}\right]\right\}$.

It turns out that Theorem 2.1 has the following extension.

Theorem 2.1*. There exists a finite constant $C_{1}^{*}$, not depending on $F$ or $h$, such that

$$
P\left\{\left\|H_{n}-H_{E}\right\|_{\infty}>d\right\} \leqq\left(1+4 C_{1}^{*} k_{\underline{n}}^{1 / 2} d\right) \exp \left\{-2 k_{\mathfrak{n}} d^{2}\right\}, \quad d>0, \underset{\sim}{n} \geqq \underset{\sim}{m} .
$$

Proof. Using an extension to generalized $U$-statistics by Serfling (1985a) of a representation given by Hoeffding (1963) for $U$-statistics, we have as an extension of (2.5) the representation

$$
H_{\sim}-H_{\sim}=\left(1 /\left[\prod_{j=1}^{c}\left(n_{j}\right) !\right]\right) \sum_{i=1}^{\prod_{j=1}^{i}\left(n_{j}\right) !}\left(H_{\sim}^{(i)}-H_{E}\right),
$$

where each $H_{a}^{(i)}$ is a classical empirical $d f$ based on $k_{\mathfrak{z}}$ i.i.d. terms having $d f H_{\mathfrak{F}}$. The remainder of the proof is analogous to that of Theorem 2.1. 
However, getting an extension of Corollary 2.1 is somewhat more complicated when $c$ may be $>1$. For this purpose, following Serfling (1985a) we introduce the following constraint on the array $\left\{\left(n_{1}, \ldots, n_{c}\right)\right\}$.

Definition 2.1. An array $\left\{\left(n_{1}, \ldots, n_{c}\right)\right\} \in \mathbb{N}^{c}$ satisfies Condition $A$ if

$$
\frac{\log \max \left(n_{1}, \ldots, n_{c}\right)}{\min \left(n_{1}, \ldots, n_{c}\right)} \rightarrow 0 \quad \text { as } \min \left(n_{1}, \ldots, n_{c}\right) \rightarrow \infty
$$

(This is trivially satisfied in the case $c=1$ and in general is not very restrictive.)

The notation " $\min \left(n_{1}, \ldots, n_{c}\right) \stackrel{(A)}{\longrightarrow} \infty$ " shall denote restriction under Condition A.

Corollary $2.1^{*}$. There exists a finite constant $C_{m}$, not depending on $\underset{\sim}{F}$ or otherwise on $h$, such that with probability 1

$$
\limsup _{\min \left(n_{1}, \ldots, n_{c}\right)-(A)}^{\longrightarrow \infty} k_{n}^{1 / 2}\left(\log n_{1} \ldots n_{c}\right)^{-1 / 2}\left\|H_{n}-H_{E}\right\|_{\infty}<C_{m} .
$$

$\left(\right.$ Here $\left.\underset{\sim}{m}=\left(m_{1}, \ldots, m_{c}\right).\right)$

The proof is a straightforward application of the Borel-Cantelli lemma.

For weighted discrepancies, the proof of Theorem 2.2 carries over to the present situation and yields.

Theorem 2.2*. Under the assumptions of Theorem 1, we have with probability 1

$$
\left\|\left(H_{\mathfrak{n}}-H_{E}\right) / q \circ H_{E}\right\|_{\infty} \rightarrow 0, \quad \min \left(n_{1}, \ldots, n_{c}\right) \stackrel{(A)}{\longrightarrow} \infty .
$$

To extend Theorem 2.3, we must restrict attention to ordered elements from the partially ordered array $\{n\}$. We define $\mu_{n}, \mu_{E}$ and $D_{n}\left(\mathbf{C}, \mu_{E}\right)$ in similar fashion as before, and we define $\mathbf{A}_{n}$ to be the $\sigma$-algebra generated by $\mathbf{A}_{n ;}^{(j)}, i \leqq j \leqq c$, where, with $\mathbf{X}_{\left(n_{j}\right)}^{(j)}=\left(X_{n_{j}, 1}^{(j)}, \ldots, X_{n_{j}, n_{j}}^{(j)}\right)$ the vector of order statistics of $X_{1}^{(j)}, \ldots, X_{n_{j}}^{(j)}$,

$$
\mathbf{A}_{n_{j}}^{(j)}=\left\{\mathbf{X}_{\left(n_{j}\right)}^{(j)}, X_{n_{j}+1}^{(j)}, X_{n_{j}+2}^{(j)}, \ldots\right\} .
$$

Then it is known (see Sen (1977), p. 288) that for fixed $B \in \mathbf{B}$, the generalized $U$-statistic array $\left\{\mu_{n}(B)-\mu_{\mathfrak{E}}(B)\right\}$ is a reverse martingale w.r.t. the $\sigma$-field $\mathbf{A}_{n}$, i.e., with probability $1, E\left\{\mu_{\underline{k}}(B)-\mu_{\mathcal{E}}(B) \mid \mathbf{A}_{n}\right\}=\mu_{n}(B)-\mu_{E}(B)$, for $\underset{\sim}{n} \geqq \underset{\sim}{k} \geqq \underset{\sim}{m}$. Hence we have

Theorem 2.3*. $\left\{D_{n}\left(C, \mu_{E}\right)\right\}$ is a reverse submartingale w.r.t. the $\sigma$-field $\mathbf{A}_{n}$, i.e., for $m \leqq k \leqq n$, with probability 1

$$
E\left\{D_{\underline{k}}\left(\mathbf{C}, \mu_{\mathcal{F}}\right) \mid A_{\mathfrak{n}}\right\} \geqq D_{\underline{n}}\left(\mathbf{C}, \mu_{\mathcal{F}}\right) .
$$

Remark 2.1. In this section we have presented certain Glivenko-Cantelli results having general interest and application. Some further a.s. results of a more specialized nature are developed in the subsequent sections of this paper; see Lemmas 3.2 and 3.3 . 


\section{A Strong Law for Generalized $L$-Statistics}

Let $H_{F}$ and $H_{n}$ be defined as previously in terms of a kernel $h: \mathbb{R}^{m} \rightarrow \mathbb{R}$ and $X_{1}, \ldots, X_{n}$ i.i.d. $F$, and let $W_{n, 1} \leqq \ldots \leqq W_{n, n_{(m)}}$ denote the ordered values of $h\left(X_{i_{1}}, \ldots, X_{i_{m}}\right)$ taken over the $n_{(m)} m$-tuples $\left(i_{1}, \ldots, i_{m}\right)$ of distinct elements from $\{1, \ldots, \mathbf{n}\}$. A wide class of parameters of $F$ can be represented usefully as $T\left(H_{F}\right)$, where $T(\cdot)$ is a functional of the general form

$$
T(G)=\int_{0}^{1} g \circ T_{t}(G) d K(t), \quad G \partial d f
$$

where $g: \mathbb{R} \rightarrow \mathbb{R}$ is Borel-measurable, $K$ is a signed measure on $(0,1)$, and for each $t$ in the support of $K, T_{t}(\cdot)$ is a classical $L$-functional: $T_{t}(G)$ $=\int_{0}^{1} G^{-1}(s) d M_{t}(s)$, where $G^{-1}(s)=\inf \{x: G(x) \geqq s\}$ and $M_{t}$ is a signed measure on $(0,1)$. This functional was introduced by Janssen et al. (1984), who established asymptotic normality results for the corresponding statistics $T\left(H_{n}\right)$ for estimation of $T\left(H_{F}\right)$.

Our purpose here is to establish strong convergence, and in fact we shall let $K(\cdot)$ in (3.1) depend on $n$, thus considering the functional

$$
T_{n}(G)=\int_{0}^{1} g \circ T_{t}(G) d K_{n}(t)
$$

We shall take the measures $d K(t), d K_{n}(t)$ and $d M_{t}(s)$ to be the differentials of

$$
\begin{array}{ll}
K(t)=\int_{0}^{t} J(u) d u+\sum_{j=1}^{d} a_{j} 1\left\{t \leqq p_{j}\right\}, & 0<p_{j}<1, j=1, \ldots, d, \\
K_{n}(t)=\int_{0}^{t} J_{n}(u) d u+\sum_{j=1}^{d} a_{j} \mathbf{1}\left\{t \leqq p_{j}\right\}, & 0<p_{j}<1, j=1, \ldots, d,
\end{array}
$$

and

$$
M_{t}(s)=\int_{0}^{s} J_{t}(u) d u+\sum_{j=1}^{d_{t}} a_{t j} 1\left\{s \leqq p_{t j}\right\}, \quad 0<p_{t j}<1, j=1, \ldots, d_{t}
$$

thus permitting both smooth and discrete weighting of quantiles $G^{-1}(s)$ in $T_{t}(G)$ and of functionals $g \circ T_{t}(G)$ in $T(\cdot)$. This covers essentially all cases of interest. Note that in the special case of (3.2) corresponding to $T_{t}(G)=G^{-1}(t)$, the statistic $T_{n}\left(H_{n}\right)$ may be expressed in the form

$$
\sum_{i=1}^{n_{(m)}} c_{n i} g\left(W_{n, i}\right)
$$


Conversely, any statistic of form (3.6) may be represented as $T_{n}\left(H_{n}\right)$ with $T_{\tau}(G)$ $=G^{-1}(t)$ and some choice of $d K_{n}(t)$ (not necessarily unique) satisfying $c_{n i}$ $=\int_{(i-1) / n_{(m)}}^{i / n_{(m)}} d K_{n}(t) ;$ e.g., take (3.4) with $d=0$ and $J_{n}(t)=n_{(m)} c_{n i}$ for $(i-1) / n_{(m)}<t$ $\leqq i / n_{(m)}, 0<t<1$. The class of statistics given by (3.6) may be termed "linear combinations of functions of generalized order statistics." For the case $g(x)=x$ it gives the "generalized $L$-statistics" (which includes both $U$-statistics and classical $L$-statistics) introduced by Serfling (1984), who established asymptotic normality results. For the special case $m=1, h(x)=x,(3.6)$ gives the class of "linear combinations of functions of order statistics", for which van Zwet (1980) proved strong convergence results under very tight conditions, confining attention to the case that $d K_{n}(t)=J_{n}(t) d t$. The present treatment extends van Zwet's by allowing limits $d K(t)$ to have a discrete component (considering $K(\cdot)$ as a limit of $K_{n}(\cdot)$ ), and considering arbitrary kernels $h$ and functionals $T_{t}(G)$ other than $G^{-1}(t)$, and retains van Zwet's general scope of allowing the transformation $g(\cdot)$ to be an arbitrary Borel-measurable function. We establish a very general and far-reaching deterministic result (Theorem 3.1), which we apply in Corollaries 3.1 and 3.2 to two broad classes of statistics: Examples 3.1, treating the general class given by (3.6), and Examples 3.2, treating some spread estimators of Bickel and Lehmann (1979).

To obtain (under appropriate assumptions) that $w p 1$

$$
T_{n}\left(H_{n}\right)-T_{n}\left(H_{F}\right) \rightarrow 0, \quad n \rightarrow \infty,
$$

and also that $T_{n}\left(H_{F}\right) \rightarrow T\left(H_{F}\right)$, we build upon the very insightful treatment of van Zwet (1980), but also separate the roles played by functional analysis and Glivenko-Cantelli theory. First we show that (3.7) holds deterministically with $H_{F}$ replaced by an arbitrary $d f G$ and $\left\{H_{n}\right\}$ replaced by an arbitrary sequence $\left\{G_{n}\right\}$ converging weakly to $G$ and satisfying other technical restrictions. This is the "functional analysis" part. Then we show that $\left\{H_{n}\right\}$ satisfies $w p 1$ the conditions imposed on $\left\{G_{n}\right\}$. This is the "Glivenko-Cantelli" part. The idea of separating the functional-analytic and probabilistic components of the strong convergence problem for $L$-statistics was introduced by Wellner (1977), who obtained a general strong law for $L$-statistics as a corollary of extended Glivenko-Cantelli theorems which he developed for the empirical $d f F_{n}$, such as we have extended in Sect. 2 to the empirical $d f^{\prime}$ 's $H_{n}$ and $H_{n}$. However, the assumptions of his theorem are not quite sharp, imposing for example $(1+\varepsilon)$-moment conditions for the sample mean. The development by van Zwet (1980) leads to an appropriately sharp strong law but entails unseparated functional-analytic and Glivenko-Cantelli components. However the separation of these components permits in a straightforward fashion important extensions to nonclassical versions of empirical $d f$ 's such as our $H_{n}$. Therefore, we take special care to achieve this separation, without sacrificing sharpness. We shall first develop the functional-analytic theory for the functional $T_{n}(G)$ given by (3.2), then treat the matter of substitution of an empirical $d f H_{n}$ or $H_{n}$.

We now state for the case of continuous $g$ our key functional-analytic theorem for the functional $T_{n}(\cdot)$ defined by (3.2), (3.4) and (3.5), and the functional $T(\cdot)$ 
given by (3.1), (3.3) and (3.5). (Extension to the general case of arbitrary Borelmeasurable $g$ requires some additional details and development, which we discuss briefly at the conclusion of Sect. 4.). The relevant functions $\left\{J_{n}(t), 0<t<1\right\}$, $\left\{J_{t}(s), 0<s<1\right\}$ for fixed $t$, and $\left\{T_{t}(G), 0<t<1\right\}$ will be viewed as functions $f:(0,1) \rightarrow \mathbb{R}$ and we shall denote the $L_{p}\left((0,1), \mathbf{B}_{(0,1)}, \lambda\right)$ norms of $f$ by $\|f\|_{p}$ $=\left(\int_{0}^{1}|f(t)|^{p} d t\right)^{1 / p}$ in the case $1 \leqq p<\infty$ and $=$ ess sup $|f|$ in the case $p=\infty$, where $\lambda$ denotes Lebesgue measure on $\left((0,1), \mathbf{B}_{(0,1)}\right)$. For the functionals $T_{t}(G), 0<t<1$, it will be convenient to use the notation $m_{G}(t)=T_{t}(G), 0<t<1$. Due to the complexity of the functional $T_{n}(G)$ as well as of the restrictions on the sequence $\left\{G_{n}\right\}$ approaching $G$, it will be convenient to state the corresponding sets of assumptions prior to the formulation of the theorem.

The conditions to be met by the functional $T_{n}(G)$ are

Assumptions (3.8). Consider the sequence of functionals $\left\{T_{n}(\cdot), n \geqq 1\right\}$ given by (3.2), (3.3) and (3.4), put $A_{0}=\bigcup_{n=1}^{\infty} A_{n}$, with $A_{n}=\left\{t: 0<t<1, J_{n}(t) \neq 0\right\}$ and $A_{t}$ $=\left\{s: 0<s<1, J_{t}(s) \neq 0\right\}, 0<t<1$, and let $G$ be a given $d f$. We assume, with $1 \leqq p$ $\leqq \infty, p^{-1}+q^{-1}=1,1 \leqq \tilde{p} \leqq \infty, \tilde{p}^{-1}+\tilde{q}^{-1}=1$, that

$$
\begin{gathered}
\left\|J_{t}\right\|_{p}<\infty, \text { each } t \in A_{0} \cup\left\{p_{1}, \ldots, p_{d}\right\} ; \\
\sup _{n}\left\|J_{n}\right\|_{p}<\infty \text { if } 1<p \leqq \infty ;
\end{gathered}
$$

or $\left\{J_{n}(\cdot), n \geqq 1\right\}$ is uniformly integrable if $p=1$;

$G^{-1}$ is continuous at $s=p_{t j}, 1 \leqq j \leqq d_{t}$, for all $t \in A_{0}$ except for $t$ in a $\lambda$-null set, and for $t=p_{1}, \ldots, p_{d}$.

For a sequence of $d f^{\prime}$ 's $\left\{G_{n}\right\}$ for which $T_{n}\left(G_{n}\right)-T_{n}(G) \rightarrow 0$ is to be established, we will require

Assumptions (3.9). Let $\left\{G_{n}\right\}$ and $G$ be $d f^{\prime}$ 's and $q, \tilde{q}, A_{0}$ and $A_{t}$ as in Assumptions (3.8). We assume $G_{n} \Rightarrow G$ and

$$
\begin{gathered}
\left\{\left(G_{n}^{-1}\right)^{\tilde{q}}, n \geqq 1\right\} \text { is uniformly integrable on } A_{t}, \\
\text { each } t \in A_{0} \cup\left\{p_{1}, \ldots, p_{d}\right\}, \text { if } 1 \leqq \tilde{q}<\infty ; \text { or } \\
\sup _{n}\left\|G_{n}^{-1} \mathbf{1}_{A_{t}}\right\|_{\infty}<\infty, \text { each } t \in A_{0} \cup\left\{p_{1}, \ldots, p_{d}\right\}, \text { if } \tilde{q}=\infty ;
\end{gathered}
$$

(3.9.b) $\left\{\left(g \circ m_{G_{n}}\right)^{q}, n \geqq 1\right\}$ is uniformly integrable on $A_{0}$ if $1 \leqq q<\infty$; or

$$
\sup _{n}\left\|\left(g \circ m_{G_{n}}\right) \mathbf{1}_{A_{0}}\right\|_{\infty} \text { if } q=\infty \text {. }
$$

Theorem 3.1. (i) Assume $g$ continuous. Suppose that the sequence of functionals $\left\{T_{n}(\cdot), n \geqq 1\right\}$ given by (3.2), (3.4) and (3.5) satisfies Assumptions (3.8) for a df $G$, and that the sequence of $d f$ 's $\left\{G_{n}\right\}$ satisfies Assumptions (3.9) for $G$. Then

$$
T_{n}\left(G_{n}\right)-T_{n}(G) \rightarrow 0, \quad n \rightarrow \infty .
$$


(ii) Suppose that the conditions of part (i) are satisfied and that there exists a function

$$
J \in L_{p}\left((0,1), \mathbf{B}_{(0,1)}, \lambda\right)
$$

such that

$$
\int_{0}^{t} J_{n}(s) d s \rightarrow \int_{0}^{t} J(s) d s, \quad \text { all } t \in(0,1)
$$

Then, for $T(\cdot)$ given by (3.1), (3.3) and (3.5) we have, assuming $\left\|\left(g \circ m_{G}\right) \mathbf{1}_{A_{0}}\right\|_{q}<\infty$,

$$
T_{n}(G) \rightarrow T(G), \quad n \rightarrow \infty .
$$

The proof of Theorem 3.1 is deferred to Sect. 4.

Turning now to the stochastic component of our development, we put $\left\{H_{n}\right\}$ and $H_{F}$ for $\left\{G_{n}\right\}$ and $G$. The main issue, then, with Assumptions (3.8) assumed with respect to $H_{F}$, is to verify that with probability $1\left\{H_{n}\right\}$ is a sequence of $d f$ 's satisfying Assumptions (3.9) and thus to conclude that $T\left(H_{n}\right) \rightarrow T\left(H_{F}\right)$ $w p 1$. Besides the probability space $\left((0,1), \mathbf{B}_{(0,1)}, \lambda\right)$ which is central to the functional-analytic treatment, we now also have a probability space $(\Omega, \mathbf{A}, \mathbb{P})$ on which our basic r.v.'s $\left\{X_{i}\right\}$ are defined. Thus we shall be speaking of almost

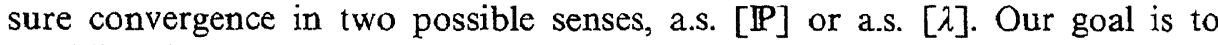
establish that $T_{n}\left(H_{n}\right) \rightarrow T\left(H_{F}\right)$ a.s. $[\mathbb{P}]$.

We shall use the property of empirical $d f$ 's that the support of $H_{n}$ must belong to that of $H_{F}$. The support of a $d f G$ is defined to be $S(G)=\{x: G(x+\varepsilon)$ $>G(x-\varepsilon)$, all $\varepsilon>0$ \}. The following is easily checked.

Lemma 3.1. If $G_{0}$ and $G_{1}$ are (right-continuous) df's with $S\left(G_{1}\right) \subset S\left(G_{0}\right)$, then

$$
G_{1}^{-1}(t)=G_{0}^{-1}\left(G_{0}\left(G_{1}^{-1}(t)\right)\right), \quad t \in(0,1) .
$$

In verifying conditions (3.9.a) or (3.9.b), it is convenient (and unrestrictive from a practical standpoint) to assume that the sets $A_{0}$ and $A_{z}, 0<t<1$, are intervals in $(0,1)$. The results we give below will be specific to the cases that the interval in question is either the open interval $(0,1)$ or (contained in) a closed interval $[a, b]$ in $(0,1)$.

Lemma 3.2. Let $1 \leqq \tilde{q} \leqq \infty$. If, for all $t \in A_{0} \cup\left\{p_{1}, \ldots, p_{d}\right\}$, either all $A_{t}=(0,1)$ and $\left\|H_{F}^{-1}\right\|_{q}<\infty$ or all $A_{t} \subset[a, b]$, then a.s. $[\mathbb{P}]$ the sequence $\left\{H_{n}\right\}$ satisfies (3.9.a); i.e., for $n$ sufficiently large $\left\{\left(H_{k}^{-1}\right)^{4}, k \geqq n\right\}$ is uniformly integrable on $A_{t}$, each $t \in A_{0} \cup\left\{p_{1}, \ldots, p_{d}\right\} \quad$ if $1 \leqq \tilde{q}<\infty$, and $\sup _{k \geq n}\left\|H_{k}^{-1} \mathbf{1}_{\boldsymbol{A}_{t}}\right\|_{\infty}<\infty$, each $t \in A_{0} \cup\left\{p_{1}, \ldots, p_{d}\right\}$ if $\tilde{q}=\infty$.

Proof. First consider the case $A_{t}=(0,1), t \in A_{0} \cup\left\{p_{1}, \ldots, p_{d}\right\}$. For $\tilde{q}=\infty$, we have by Lemma 3.1 that

$$
\left\|H_{n}^{-1}\right\|_{\infty}=\sup _{t}\left|H_{n}^{-1}(t)\right|=\sup _{t}\left|H_{F}^{-1} \circ H_{F} \circ H_{n}^{-1}(t)\right| \leqq\left\|H_{F}^{-1}\right\|_{\infty} .
$$


For $\tilde{q}<\infty$, we have

$$
\left\|H_{n}^{-1}\right\| \|_{\frac{q}{q}}=\int_{0}^{1}\left|H_{n}^{-1}(t)\right|^{q} d t=\int_{\mathbb{R}}|y|^{q} d H_{n}(y)=n_{(m)}^{-1} \sum\left|h\left(X_{i_{1}}, \ldots, X_{i_{m}}\right)\right|^{q},
$$

so that $\left\|H_{n}^{-1}\right\|_{\bar{q}}^{\bar{q}}$ is a $U$-statistic with kernel $|h|^{\natural}$ and a.s. $[\mathbb{P}]$ converges to $\left\|H_{F}^{-1}\right\|_{q}^{q}<\infty$ by the SLLN for $U$-statistics. Hence, by a standard result, a.s. $[\mathbb{P}]$ the functions $\left\{\left(H_{k}^{-1}\right)^{\natural}, k \geqq n\right\}$ are uniformly integrable on $(0,1)$, for $n$ sufficiently large.

If $A_{t} \subset[a, b]$, for all $t \in A_{0} \cup\left\{p_{1}, \ldots, p_{d}\right\}$, take $0<a-\varepsilon, b+\varepsilon<1$ continuity points of $H_{F}^{-1}$. Then a.s. $[\mathbb{P}] \sup _{t}\left|H_{n}^{-1}(t) 1_{A_{t}}\right| \leqq\left|H_{F}^{-1}(a-\varepsilon)\right|+\left|H_{F}^{-1}(b+\varepsilon)\right|$ for all $n$ sufficiently large. Therefore in this case (3.9.a) is immediate.

Verification of (3.9.b) in general form is rather troublesome, but in practice it can be checked ad hoc for typical cases of the functionals $\left\{T_{r}(G), t \in A_{0}\right\}$, under effective restrictions on $g$ and $A_{0}$ which do not preclude the motivating applications for this investigation. For such purposes the following result is useful.

Lemma 3.3. Assume $g$ continuous. Suppose that (3.8.a), (3.8.c) and the conditions of Lemma 3.2 are satisfied. Assume that a.s. $[\mathbb{P}]$

$$
\underset{n \rightarrow \infty}{\limsup } \sup _{t \in A_{0}}\left|T_{t}\left(H_{n}\right)\right|<\infty \text {. }
$$

Let $1 \leqq q \leqq \infty$ be given. Then a.s. $[\mathbb{P}]$ the sequence $\left\{H_{n}\right\}$ satisfies (3.9.b); i.e., for $n$ sufficiently large $\left\{\left(g \circ m_{H_{k}}\right)^{q}, k \geqq n\right\}$ is uniformly integrable on $A_{0}$ if $1 \leqq q<\infty$; or $\sup _{k \geqq n}\left\|\left(g \circ m_{H_{k}}\right) \mathbf{1}_{A_{0}}\right\|_{\infty}<\infty$ if $q=\infty$.

Proof. We have a.s. $[\mathbb{P}]$

$$
\left\|\left(g \circ m_{B_{n}}\right) \mathbf{1}_{A_{0}}\right\|_{\infty} \leqq \sup _{t \in A_{0}}\left|g \circ T_{t}\left(H_{n}\right)\right| \leqq \sup _{x \in B\left(H_{n}\right)}|g(x)|
$$

where $B\left(H_{n}\right)=\left\{T_{t}\left(H_{n}\right), t \in A_{0}\right\}$. By (3.15), for all but a finite number of $n$ 's, $B\left(H_{n}\right)$ is contained in a compact set, whence by continuity of $g$ the term on the right in (3.16) is bounded for $n$ sufficiently large. Thus for $q=\infty$ the conclusion of the lemma follows from (3.16). If $1 \leqq q<\infty$ (3.16) implies for $\varepsilon>0$ a.s. [ $\mathbb{P}]$ boundedness of $\left\|\left(g \circ H_{n}^{-1}\right) \mathbf{1}_{A_{0}}\right\|_{q(1+\varepsilon)}$ for $n$ sufficiently large. This is sufficient for the desired uniform integrability.

With these tools, we now can give two very general convergence results. We first deal in Corollary 3.1 with strong convergence of $T_{n}\left(H_{n}\right)$, with $T_{n}(\cdot)$ given by (3.2), for the important special case $T_{t}(G)=G^{-1}(t)$. Secondly, Corollary 3.2 deals with the strong convergence of $T_{n}\left(H_{n}\right)$, with $T_{t}(G)$ an $L$-functional. Both results are specializations of Theorem 3.1 to the stochastic sequence $\left\{H_{n}\right\}$.

Note also, that, with $H_{F}$ and $\left\{H_{n}, n \geqq 1\right\}$ for $G$ and $\left\{\mathrm{G}_{n}, \mathrm{n} \geqq 1\right\}$, we have by our Glivenko-Cantelli result (Corollary 2.1) that $H_{n} \Rightarrow H_{F}$ a.s. $[\mathbb{P}]$. 
Corollary 3.1. Assume $g$ continuous. Let $T(\cdot)$ and $\left\{T_{n}(\cdot), n \geqq 1\right\}$ be given by (3.1) and (3.2) with $T_{t}(G)=G^{-1}(t)$ and $d K(t), d K_{n}(t)$ as in (3.3) and (3.4). Suppose $\left\{J_{n}(\cdot), n \geqq 1\right\}$ satisfies (3.8.b), $H_{F}$ satisfies (3.8.c) and that either $A_{0}=(0,1)$ and $\left\|g \circ H_{F}^{-1}\right\|_{q}<\infty$ or $A_{0} \subset\left[a_{0}, b_{0}\right] \subset(0,1)$, where $1 \leqq p \leqq \infty, p^{-1}+q^{-1}=1$. Also let $J(\cdot)$ be determined by (3.11) and (3.12). Then a.s. $[\mathbb{P}]$

$$
T_{n}\left(H_{n}\right) \rightarrow T\left(H_{F}\right), \quad n \rightarrow \infty .
$$

Proof. To apply Theorem 3.1, we verify that Assumptions (3.8) and (3.9) hold a.s. $[\mathbb{P}]$ for $\left\{H_{n}\right\}$. Since $T_{t}(G)=G^{-1}(t)$, we have $J_{t}(s)=0$, each $t \in A_{0} \cup\left\{p_{1}, \ldots, p_{d}\right\}$, so $A_{t}=\phi$ and (3.8.a) and (3.9.a) are vacuous. Since, $m_{G}=G^{-1}$, (3.9.b) simply means that a.s. $[\mathbb{P}]\left\{\left(g \circ H_{k}^{-1}\right)^{q}, k \geqq n\right\}$ is uniformly integrable on $A_{0}$ for $n$ sufficiently large if $1 \leqq q<\infty$; or $\sup _{k \geqq n}\left\|\left(g \circ H_{k}^{-1}\right) 1_{A_{0}}\right\|_{\infty}<\infty$ if $q=\infty$. For the case $A_{0}=(0,1),(3.9 . b)$ follows by repeating the first part of the proof of Lemma 3.2 with $H_{n}^{-1}$ replaced by $g \circ H_{n}^{-1}$ and $H_{F}^{-1}$ replaced by $g \circ H_{F}^{-1}$. If $A_{0} \subset\left[a_{0}, b_{0}\right]$ we argue as in the proof of Lemma 3.3.

Remark 3.1. Note that our corollaries remain valid if we take $A_{0}=\bigcup_{n=N}^{\infty} A_{n}$ for some $N \in \mathbb{N}$.

\section{Examples 3.1}

Linear combinations of functions of generalized order statistics. Corollary 3.1 essentially covers the class of statistics (3.6), which includes some classical collections of statistics as well as new varieties of recent interest in the literature. We present several examples.

(i) Linear combinations of functions of order statistics. Here $m=1, h(x)=x$. For this specific choice and taking $A_{0}=(0,1)$ Corollary 3.1 reduces to Corollary 2.1 of van Zwet (1980), specialized to the case $g$ continuous. (Following the proof of Theorem 3.1, in Sect. 4, we discuss extension to the case of arbitrary Borel-measurable functions $g(\cdot)$.) Confining attention to the case $g(x)=x$, we mention the sample mean (take $J_{n}(t) \equiv 1, d=0$, and assume $\left\|F^{-1}\right\|_{1}<\infty$, i.e., $F$ has finite mean), the sample median (take $J_{n}(t) \equiv 0, d=1=a_{1}, p_{1}=\frac{1}{2}$, and assume that $F^{-1}$ is continuous at $\frac{1}{2}$, i.e., that $\frac{1}{2}$ is the unique solution of $F(x-) \leqq \frac{1}{2} \leqq F(x)$ ), trimmed means $\left(J_{n}(t)=(n /(n-2[\alpha n])) \mathbf{1}\{[\alpha n] / n \leqq t \leqq(n-[\alpha n]) / n\}, d=0\right)$, Gini's mean difference $\left(J_{n}(t)=(n /(n-1))(4 t-2), d=0\right.$ and assume $\left.\left\|F^{-1}\right\|_{1}<\infty\right)$, etc.

(ii) $U$-statistics. Apply Corollary 3.1 with $g(x)=x, J_{n}(t) \equiv 1, d=0$, assuming $\left\|H_{F}^{-1}\right\|_{1}<\infty$, i.e., $E|h|<\infty$. Hence the corollary reduces to the strong law of large numbers for $U$-statistics, obtained by Hoeffding (1961).

(iii) Trimmed $U$-statistics. Apply Corollary 3.1 with $g(x)=x$,

$$
\left.J_{n}(t)=\left(n_{(m)}\right)\left(n_{(m)}-2\left[\alpha n_{(m)}\right]\right)\right) \mathbf{1}\left\{\left[\alpha n_{(m)}\right] \leqq n_{(m)} t \leqq n_{(m)}-\left[\alpha n_{(m)}\right]\right\}, \quad d=0 .
$$

In particular, a "trimmed variance" is given by taking $m=2, h\left(x_{1}, x_{2}\right)=\frac{1}{2}\left(x_{1}\right.$ $\left.-x_{2}\right)^{2}$. 
(iv) Another spread measure. Apply Corollary 3.1 with $g(x)=x, J_{n}(t) \equiv 0, d=1$ $=a_{1}, p_{1}=\frac{1}{2}$, and $m=2, h\left(x_{1}, x_{2}\right)=\left|x_{1}-x_{2}\right|$. This yields a spread measure considered by Bickel and Lehmann (1979), the relevant parameter being the median of the distribution of $\left|X_{1}-X_{2}\right|$.

(v) Generalized Hodges-Lehmann location estimators. Apply Corollary 3.1 with $g(x)=x, J_{n}(t) \equiv 0, d=1=a_{1}$ and $h\left(x_{1}, \ldots, x_{m}\right)=m^{-1}\left(x_{1}+\ldots+x_{m}\right)$. (For $m=1$ this gives the sample median and for $m=2$ the classical Hodges-Lehmann estimator.).

Corollary 3.2. Assume $g$ continuous. Let $T(\cdot)$ and $\left\{T_{n}(\cdot), n \geqq 1\right\}$ be given by (3.1) and (3.2) with $d K(t), d K_{n}(t)$ and $d M_{t}(s)$ as in (3.3)-(3.5). Suppose that the weight functions satisfy (3.8.a) and (3.8.b) and that $H_{F}$ satisfies (3.8.c), and $\left\|\left(g \circ m_{H_{F}}\right) \mathbf{1}_{A_{0}}\right\|_{q}<\infty$. Further suppose for all $t \in A_{0} \cup\left\{p_{1}, \ldots, p_{d}\right\}$ either $A_{t}=(0,1)$ and $\left\|H_{\vec{p}}^{-1}\right\|_{\bar{q}}<\infty$ or $A_{t} \subset[a, b]$; where $1 \leqq p \leqq \infty, p^{-1}+q^{-1}=1,1 \leqq \tilde{p} \leqq \infty, \tilde{p}^{-1}$ $+\tilde{q}^{-1}=1$. If a.s. $[\mathbb{P}] T_{t}\left(H_{n}\right), t \in A_{0}$, satisfies (3.15), then a.s. $[\mathbb{P}]$

$$
T_{n}\left(H_{n}\right) \rightarrow T\left(H_{F}\right), \quad n \rightarrow \infty .
$$

Proof. We only have to verify that Assumptions (3.9) hold a.s. $[\mathbb{P}]$ for $\left\{H_{n}\right\}$. For (3.9.a) we use Lemma 3.2 and (3.9.b) is immediate from Lemma 3.3.

\section{Examples 3.2}

Some spread estimators of Bickel and Lehmann. We consider here some functionals $T(\cdot)$ for which $T\left(H_{n}\right)$ gives statistics of the type proposed by Bickel and Lehmann (1979) as measures of spread for a $d f F$ not necessarily symmetric about any known point, and for which asymptotic normality has been proved by Janssen, Serfling and Veraverbeke (1984).

(i) Consider the functional

$$
T(F)=\int_{0}^{1}\left|F^{-1}(t)-F^{-1}(1-t)\right|^{\gamma} d K(t) .
$$

Bickel and Lehmann (1979) suggest the case $\gamma=2$ and $K(\cdot)$ uniform on $[\beta, 1-\beta]$, where $0<\beta<\frac{1}{2}$, giving

$$
T_{\beta}(F)=(1-2 \beta)^{-1} \int_{\beta}^{1-\beta}\left[F^{-1}(t)-F^{-1}(1-t)\right]^{2} d t .
$$

The corresponding statistic is given by

$$
T_{\beta n}\left(F_{n}\right)=(n-2[\beta n])^{-1} \sum_{k=[\beta n]+1}^{n-[\beta n]}\left(X_{n, k}-X_{n, n-k}\right)^{2} .
$$

Then (3.20) is of form (3.2) with the specializations $h(x)=x$ (making $H_{F}=F$ and $\left.H_{n}=F_{n}\right) ; \quad g(x)=x^{2} ; \quad J_{n}(t)=(n /(n-2[\beta n])) \mathbf{1}\{[\beta n] / n \leqq t \leqq(n-[\beta n]) / n\} ;$ 
$A_{0} \subset[\beta-\eta, 1-\beta+\eta] \subset(0,1)$ for some $\eta>0 ; d=0$; and for each $t \in A_{0}, J_{t}(s)=0$, $A_{t}=\phi, d_{t}=2, a_{t 1}=1=-a_{t 2}, p_{t 1}=t=1-p_{t 2}$, i.e., $T_{t}(G)=G^{-1}(t)-G^{-1}(1-t)$. To apply Corollary 3.2 we take $p=\infty, q=1$ and need only verify the assumptions $\left\|m_{F} \mathbf{1}_{[\beta-\eta, 1-\beta+\eta]}\right\|_{1}<\infty$ and (3.15).

Now in the present case $m_{F}(t)=F^{-1}(t)-F^{-1}(1-t)$, and we trivially have

$$
\left\|m_{F} \mathbf{1}_{[\beta-\eta, 1-\beta+\eta]}\right\|_{1} \leqq 2\left(\left|F^{-1}(\beta-\eta)\right|+\left|F^{-1}(1-\beta+\eta)\right|\right)<\infty .
$$

Condition (3.15) reduces to a.s. $[\mathbb{P}]$ that

$$
\limsup _{n \rightarrow \infty} \sup _{t \in[\beta-\eta, 1-\beta+\eta]}\left|F_{n}^{-1}(t)-F_{n}^{-1}(1-t)\right|<\infty,
$$

which follows easily by arguments used above. Finally note that the difference between

$$
\Upsilon_{\beta n}=(n-2[\beta n])^{-1} \sum_{k=[\beta n]+1}^{n-[\beta n]}\left(X_{n, k}-X_{n, n-k+1}\right)^{2}
$$

the spread estimator for (3.19) proposed by Bickel and Lehmann (1979), and $T_{\beta n}\left(F_{n}\right)$ tends a.s. to zero. Therefore, we obtain, without any assumption on $F$, that a.s. $[\mathbb{P P}] T_{\beta n} \rightarrow T(F), n \rightarrow \infty$.

(ii) Consider the functional

$$
T_{\alpha \beta}(G)=\left(\frac{1}{1-\alpha-\beta}\right) \int_{\alpha}^{1-\beta}\left[G^{-1}\left(\frac{t+1}{2}\right)\right]^{2} d t,
$$

where $0<\alpha<\frac{1}{2}<1-\beta<1$. Bickel and Lehmann (1979) introduced another spread statistic which is given by $T_{\alpha \beta}\left(H_{n}\right)$, for the kernel $h\left(x_{1}, x_{2}\right)=x_{1}-x_{2}$. In this case $H_{F}$ is the symmetric (about 0$) d f$ of $X-X^{\prime}$, for $X$ and $X^{\prime}$ independent r.v.'s with $d f F$. The functional $T_{\alpha \beta}(\cdot)$ is of form (3.1) with $g(x)=x^{2}, J(t)$ $=\mathbf{1}_{A_{0}}(t) /(1-\alpha-\beta), A_{0}=[\alpha, 1-\beta], d=0$; and for each $t \in A_{0}, J_{t}(s) \equiv 0, A_{t}=\phi$, $d_{t 1}=1=a_{t 1}, p_{t 1}=\frac{1}{2}(t+1)$, i.e., $T_{t}(G)=G^{-1}\left(\frac{t+1}{2}\right)$. To apply Corollary 3.2, we take $p=\infty, q=1$ and we have

$$
\left\|m_{H_{F}} \mathbf{1}_{[\alpha, 1-\beta]}\right\|_{1}=\left\|H_{F}^{-1}\left(\frac{t+1}{2}\right) \mathbf{1}_{[\alpha, 1-\beta]}\right\|_{1} \leqq\left|H_{F}^{-1}\left(\frac{\alpha+1}{2}\right)\right|+\left|H_{F}^{-1}\left(1-\frac{\beta}{2}\right)\right|<\infty .
$$

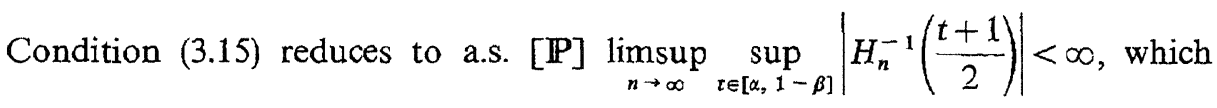
again follows by previously used arguments. Thus we conclude, without any assumptions on $F$ or $H_{F}$, that a.s. $[\mathbb{P}] T_{\alpha \beta}\left(H_{n}\right) \rightarrow T_{\alpha \beta}\left(H_{F}\right), n \rightarrow \infty$.

Extensions to the multi-sample case are straightforward, now that the functional-analytic and "Glivenko-Cantelli" parts of the problem have been separated. The relevant basic Glivenko-Cantelli theorem giving $\left\|H_{n}-H_{F}\right\| \rightarrow 0$, and 
thus $H_{n} \Rightarrow H_{E}$ a.s. $[\mathbb{P}]$, is available in Corollary $2.1^{*}$ of Sect. 2. The SLLN for $U$-statistics ussed in Lemma 3.2 becomes replaced by the one for "generalized" $U$-statistics (Sen (1977) and McConnell (1987)). Introducing other appropriate notions regarding convergence with multi-dimensional indices, and imposing appropriate restrictions, one can obtain for the functional given by (3.1) that a.s. $[\mathbb{P P}] T\left(H_{n}\right) \rightarrow T\left(H_{E}\right)$ as $\min \left(n_{1}, \ldots, n_{c}\right) \stackrel{(A)}{\longrightarrow} \infty$. The detailed treatment of the multi-sample case will be pursued elsewhere.

\section{Proof and Extensions}

In the proof of Theorem 3.1 the following basic convergence lemma will be instrumental.

Lemma 4.1. Let $1 \leqq r \leqq \infty$ and $r^{-1}+s^{-1}=1$. Let the r.v.'s $\xi$, $\left\{\xi_{n}\right\}$ and $\left\{\eta_{n}\right\}$ on a probability space $(\Omega, \mathbf{A}, \mathbb{P})$ satisfy $\xi_{n} \stackrel{p}{\longrightarrow} \xi$ and either

(i) $1 \leqq r<\infty,\left\{\left|\xi_{n}\right| r\right\}$ is uniformly integrable and $\sup _{n}\left\|\eta_{n}\right\|_{s}<\infty$,

or

(ii) $r=\infty, \sup _{n}\left\|\xi_{n}\right\|_{\infty}<\infty$ and $\left\{\eta_{n}\right\}$ is uniformly integrable.

Then $\left\|\left(\xi_{n}-\xi\right) \eta_{n}\right\|_{1} \rightarrow 0, n \rightarrow \infty$.

Proof of Theorem 3.1. We first consider convergence of $m_{G_{n}}(t)=T_{t}\left(G_{n}\right), n \rightarrow \infty$, for a fixed $t \in A_{0} \cup\left\{p_{1}, \ldots, p_{d}\right\}$. Note that

$$
m_{G_{n}}(t)=\int_{0}^{1} G_{n}^{-1}(s) J_{t}(s) d s+\sum_{j=1}^{d_{t}} a_{t j} G_{n}^{-1}\left(p_{t j}\right)
$$

Note that the convergence $G_{n} \Rightarrow G$ implies (see Lemma 1.5.6 of Serfling (1980) and its proof) that

$$
G_{n}^{-1}(t) \rightarrow G^{-1}(t), \quad \text { all continuity points } t \text { of } G^{-1},
$$

and hence

$$
\lambda\left\{t: G_{n}^{-1}(t) \rightarrow G^{-1}(t), n \rightarrow \infty\right\}=0,
$$

i.e., $G_{n}^{-1}$ converges to $G^{-1}$ a.s. [ $\left.\lambda\right]$ and hence in $\lambda$-measure. We apply Lemma 4.1 with $(\Omega, \mathbf{A}, \mathbb{P})=\left((0,1), \mathbf{B}_{(0,1)}, \lambda\right)$ and $\xi=G^{-1} \mathbf{1}_{A_{t}}, \xi_{n}=G_{n}^{-1} \mathbf{1}_{A_{t}}, \eta_{n}=J_{t}, r=\tilde{q}$, $s=\tilde{p}$. The hypotheses of the lemma are verified using (3.8.a), (3.9.a) and (4.1). This yields

$$
\int_{0}^{1} G_{n}^{-1}(s) J_{t}(s) d s \rightarrow \int_{0}^{1} G^{-1}(s) J_{t}(s) d s
$$


for all $t \in A_{0} \cup\left\{p_{1}, \ldots, p_{d}\right\}$. The same is true, by (3.8.c) and (4.1.a) for the other part of $T_{t}\left(G_{n}\right)$ and hence also for $T_{t}\left(G_{n}\right)$ itself for all $t \in A_{0} \cup\left\{p_{1}, \ldots, p_{d}\right\}$, except for $t$ in a $\lambda$-null set not including $\left\{p_{1}, \ldots, p_{d}\right\}$. Thus we have proved that for $t=p_{1}, \ldots, p_{d}$ and almost all $t$ in $A_{0}$,

$$
m_{G_{n}}(t) \rightarrow m_{G}(t)
$$

Whence, by the continuity of $g$,

$$
g \circ m_{G_{n}} \rightarrow g \circ m_{G} \quad \text { in } \lambda \text {-measure. }
$$

Now we apply Lemma 4.1 again, with the same probability space as above but $\xi=\left(g \circ m_{G}\right) \mathbf{1}_{A_{0}}, \xi_{n}=\left(g \circ m_{G_{n}}\right) \mathbf{1}_{A_{0}}, \eta_{n}=J_{n}, r=q, s=p$. The hypotheses of the lemma are verified using (3.8.b), (3.9.b) and (4.3), and we thus have proved

$$
\int_{0}^{1}\left[g \circ T_{t}\left(G_{n}\right)-g \circ T_{t}(G)\right] J_{n}(t) d t \rightarrow 0, \quad n \rightarrow \infty .
$$

Applying continuity of $g$ in connection with (4.2) for $t=p_{1}, \ldots, p_{d}$, and combining with (4.4), we obtain (3.10), completing the proof of part (i) of the theorem.

Regarding part (ii) we use the assumptions (3.8.b), (3.11), (3.12) and the fact that $g \circ T_{t}(G)$ is assumed to be in $L_{q}$ in combination with Sects. IV.8.11, IV,13.23, IV.13.25 and IV.13.27 of Dunford and Schwartz (1958) to obtain

$$
\int_{0}^{1} g \circ T_{t}(G) J_{n}(t) d t \rightarrow \int_{0}^{1} g \circ T_{t}(G) J(t) d t, \quad n \rightarrow \infty,
$$

and hence (3.13).

Remark 4.1. (i) Theorem 3.1 is a deterministic version and generalization, for $g$ continuous, of Theorem 2.1 and Corollary 2.1 of van Zwet (1980).

(ii) As mentioned already in Example 3.1(i), our Corollary 3.1 with $m=1$, $h(x)=x$ and $A_{0}=(0,1)$ reduces to Corollary 2.1 of van Zwet (1980), except that for simplicity of presentation we have restricted $g$ to be continuous (note that we do not require $H_{F}^{-1}$ to be continuous). Following van Zwet's approach in spirit this condition can be removed by an application of Luzin's theorem, giving the following extension.

Corollary 4.1. Corollary 3.1 remains valid for arbitrary Borel-measurable g satisfying

$$
g \text { is continuous at } H_{F}^{-1}\left(p_{j}\right), \quad j=1, \ldots, d
$$

and, for the case $A_{0} \subset\left[a_{0}, b_{0}\right] \subset(0,1)$,

$$
g \text { is bounded on }\left[H_{F}^{-1}\left(a_{0}\right)-\varepsilon, H_{F}^{-1}\left(b_{0}\right)+\varepsilon\right] \text {, for some } \varepsilon>0 \text {. }
$$

Proof. To prove Corollary 3.1 the continuity of $g$ is used to show, in the case $T_{t}(G)=G^{-1}(t)$, the validity of (4.3) and the boundedness of the r.h.s. in (3.17). 
The latter is still valid by (4.6). To show that (4.3) is still valid, it suffices to prove that a.s. $[\mathbb{P}]$

$$
\lim _{n \rightarrow \infty} \lambda\left\{t \in A_{0}:\left|g \circ H_{n}^{-1}(t)-g \circ H_{F}^{-1}(t)\right|>\delta\right\}=0, \quad \text { every } \delta>0 .
$$

By Luzin's theorem we have for any $\varepsilon>0$ the existence of a Borel set $B$ and a continuous function $\tilde{g}$ with $H_{F}(B)<\varepsilon$ and such that $g=\tilde{g}$ on $\mathbb{R} \backslash B$. Therefore, with $B_{n}=\left\{t: H_{n}^{-1}(t) \in B\right\}$,

$$
\begin{aligned}
& \underset{n \rightarrow \infty}{\limsup } \lambda\left\{t \in A_{0}:\left|g \circ H_{n}^{-1}(t)-g \circ H_{F}^{-1}(t)\right|>\delta\right\} \leqq H_{F}(B)+\underset{n \rightarrow \infty}{\limsup } H_{n}(B) \\
& \quad+\underset{n \rightarrow \infty}{\limsup } \lambda\left\{t \in A_{0}:\left|\tilde{g} \circ H_{n}^{-1}(t)-\tilde{g} \circ H_{F}^{-1}(t)\right|>\delta\right\} .
\end{aligned}
$$

Since $H_{n}^{-1}(t) \rightarrow H_{F}^{-1}(t), n \rightarrow \infty$, a.s. $[\mathbb{P}]$ for all continuity points $t$ of $H_{F}^{-1}(\cdot)$, the last term in the r.h.s. of (4.6) equals zero. The sum of the first two terms is less than $2 \varepsilon$. Hence the proof is complete.

(iii) We finally note that our general deterministic result, Theorem 3.1, can also be stated without the continuity assumption on $g$. This development involves formulation of additional convergence concepts for sequences $\left\{G_{n}, n\right.$ $\geqq 1\}$ and is omitted for brevity here. Complete details can be found in Helmers et al. (1985) and Serfling (1985b).

\section{References}

Bickel, P.J., Lehmann, E.L.: Descriptive statistics for non-parametric models. IV. Spread. In: Jurečková, J. (ed.) Contributions to statistics. Hájek Memorial Volume, pp. 33-40. Prague: Academia 1979

Chung, K.L.: An estimate concerning the Kolmogorov limit distribution. Trans. Am. Math. Soc. 67, 36-50 (1949)

Dunford, N., Schwartz, J.T.: Linear operators, vol. I. New York: Wiley 1958

Dvoretzky, A., Kiefer, J., Wolfowitz, J.: Asymptotic minimax character of the sample distribution function and of the classical multinomial estimator. Ann. Math. Statist. 27, 642-669 (1956)

Gaenssler, P.: Empirical processes. Hayward, Calif.: Institute of Mathematical Statistics, Lecture Notes-Monograph Series, vol. 3 (1983)

Helmers, R: Edgeworth expansions for linear combinations of order statistics. Matbematical Centre Tracts, vol. 105. Amsterdam: Mathematisch Centrum 1982

Helmers, R., Janssen, P., Serfling, R.: Glivenko-Cantelli properties of some generalized empirical $d f$ 's and strong convergence of generalized L-statistics. Technical Report No. 460, Dept. of Math. Sciences, Johns Hopkins University, Baltimore (1985)

Hoeffding, W.: The strong law of large numbers for $U$-statistics. Univ. of North Carolina Institute of Statistics Mimeo Series, No. 302 (1961)

Hoeffding, W.: Probability inequalities for sums of bounded random variables. J. Am. Stat. Assoc. 58, 13-30 (1963)

Janssen, P., Serfling, R, Veraverbeke, N.: Asymptotic normality for a general class of statistical functions and application to measure of spread. Ann. Stat. 12, 1369-1379 (1984)

McConnell, T.R.: Two-parameter strong laws and maximal inequalities for U-statistics. Proc. of the Royal Soc. of Edinburgh 107 A, 133-151 (1987)

Sen, P.K.: Almost sure convergence of generalized $U$-statistics. Ann. Probab. 5, 287-290 (1977)

Serfling, R.J.: Approximation theorems of mathematical statistics. New York: Wiley 1980 
Serfling, R.J.: Generalized $L-, M$ - and $R$-statistics. Ann. Stat. 12, 76-86 (1984)

Serfling, R.J.: A Bahadur representation for quantiles of empirical $d f$ 's of generalized $U$-statistic structure. Technical Report No. 453, Dept. of Math. Sciences, Johns Hopkins University, Baltimore (1985a)

Serfling, R.J.: A note on convergence of functions of random elements. Technical Report No. 459, Dept. of Math. Sciences, Johns Hopkins University, Baltimore (1985b)

$\operatorname{van}$ Zwet, W.R.: A strong law for linear functions of order statistics. Ann. Probab. 8, 986-990 (1980)

Wellner, J.A.: A Glivenko-Cantelli theorem and strong laws of large numbers for functions of order statistics. Ann. Stat. 5, 473-480 (1977). Correction, ibid. 6, 1394 (1977)

Received February 24, 1986; in revised form March 5, 1988 